\title{
Experimental method for developing coordination abilities in 10-14-year-old children engaged in hand-to-hand combat
}

\author{
Vladimir Sklizkov ${ }^{1}$, Albert Baimurzin ${ }^{2 *}$ \\ ${ }^{1}$ Russian State University of Physical Culture, Sport, Youth and Tourism, 105122, Moscow, Russia \\ ${ }^{2}$ Moscow State University of Food Production, 125080, Moscow, Russia
}

\begin{abstract}
The article is devoted to the study of an experimental method for the coordination abilities development based on the rhythm sense development. The study revealed the relationship between the level of students physical and technical readiness with the level of their rhythm sense development, rhythm dependence of movement skills during hand-to-hand combat, the ability of training aimed at developing a sense of rhythm, to include an auditory analyzer in the work of students. The study showed the effectiveness of the experimental method.
\end{abstract}

\section{Introduction}

Hand-to-hand combat (HC) places very high demands on the level of fighters' coordination training. The development of coordination abilities (CA) is one of the most important tasks in HC classes. The adopted program of classes in HC mainly solves the problems of developing students' CA. But it has a lack of one the most important coordination abilities development - a sense of rhythm. The program needs to be updated [1,2].

The experimental method of CA development in children aged 10-14 years engaged in HC is based on development of rhythm sense in HC classes.

The essence of the technique is that all exercises during HC classes are performed in an externally set rhythm. The coach sets the rhythm with his voice, claps, using an electronic metronome or music with a pronounced rhythm.

\section{Materials and methods}

Research methods:

1. Analysis of scientific and methodological literature.

2. Conversation, interviewing.

3. Pedagogical observations.

3. Control tests (testing).

4. Pedagogical experiment.

5. Mathematical and statistical methods of processing the obtained data.

To conduct a pedagogical experiment, two groups of 13 children, an experimental and a control group, were formed from children aged 10-14 who were engaged in HC in the Health club in Klin, Russia. Children in groups were selected by age, period of training, level of physical development and physical fitness in such a way as to achieve maximum similarity of groups. The average age in the groups was $11.6 \pm 0.35$ years in the experimental group (EG) and $12.0 \pm 0.35$ years in the control group (CG). HC classes in both groups were conducted in the same way, with one exception, in the EG all exercises were performed in a given rhythm $[1,2,3,4]$.

Before starting the experiment, both groups were tested for the level of physical qualities and CA development. The level of physical fitness was assessed by tests from the standard program for HC [5]. The test results are shown in table 1.

Table1. Results of testing the level of general physical fitness before the beginning of the pedagogical experiment

\begin{tabular}{|l|c|c|c|}
\hline \multicolumn{1}{|c|}{ Tests } & EG & CG & p \\
\hline $\begin{array}{l}\text { Flexion and extension of the arms, lying } \\
\text { down, number of times }\end{array}$ & $28,5 \pm 3,5$ & $30,1 \pm 2,4$ & $>0,05$ \\
\hline Pull-up on the crossbar, number of times & $7,6 \pm 1,5$ & $7,0 \pm 1,1$ & $>0,05$ \\
\hline $\begin{array}{l}\text { Lifting the body from the supine position, } \\
\text { number of times }\end{array}$ & $35 \pm 2,7$ & $34 \pm 2,8$ & $>0,05$ \\
\hline
\end{tabular}

\footnotetext{
${ }^{*}$ Corresponding author: miit511@ mail.ru
} 


\begin{tabular}{|l|c|c|c|}
\hline Long jump from a standstill, cm & $176 \pm 6,1$ & $178 \pm 6,5$ & $>0,05$ \\
\hline Running $30 \mathrm{~m}, \mathrm{~s}$ & $5,58 \pm 0,13$ & $5,81 \pm 0,13$ & $>0,05$ \\
\hline
\end{tabular}

The table shows that there is no statistically significant difference between the groups.

The level of CA development was evaluated using the standard tests for V. I. Lyakh [3,4,5]: "Shuttle run 3x10m" and "Three somersaults forward", tests specially selected for testing children engaged in HC, this is: "Shuttle run $4 \times 10 \mathrm{~m}$ with cones», "Cross", "Passing the ball from the wall".

"Shuttle run 4x10m with cones", is a modified version of the "Shuttle run $4 \times 9 \mathrm{~m}$ " test developed to evaluate the performance of hockey players [3]. It is performed as follows. Cones are installed at a distance of $10 \mathrm{~m}$ from each other. The subject starts from one of the cones and holds three hollow cones in his hands. At the command "March" begins running around the cones. During the flow around the cone, the subject must put on the streamlined cone, one of the cones in his hands. If it doesn't hit the cone or the cones fall, the race attempt doesn't count. The run time is measured with an accuracy of at least 0.1 seconds.

The "cross" test is aimed at identifying the ability to rearrange movements and speed $[3,6,7,8]$. A cross is drawn on the carpet using a plaster. The subject stands in one of the corners. His task is to jump, feet together, at the corners of the cross, first clockwise, then back. A total of 12 jumps are performed in each direction. The time for performing jumps in each direction is determined independently, with an accuracy of at least 0.1 seconds. then the results are summed up.

"Passing the ball from the wall" this test evaluates accuracy and speed [2,3,5]. The test is performed as follows. The subject stands at a distance of $2 \mathrm{~m}$ from the wall, holding a small basketball in his hands. The task is to throw the ball into the wall for 30 seconds and catch it. The number of transfers completed in 30 seconds is calculated.

According to the results of CA testing, table 2, there were no statistically significant differences between the groups.

Table 2. Results of testing coordination abilities before the beginning of the pedagogical experiment.

\begin{tabular}{|l|c|c|c|}
\hline \multicolumn{1}{|c|}{ Tests } & EG & CG \\
\hline Rhythm sense, conventional unit & $735 \pm 21,7$ & $723 \pm 23,9$ & $>0,05$ \\
\hline "Cross", s & $10,21 \pm 0,32$ & $10,32 \pm 0,28$ & $>0,05$ \\
\hline $\begin{array}{l}\text { Passing the ball from the wall, number of } \\
\text { times }\end{array}$ & $29,5 \pm 1,9$ & $30,45 \pm 1,56$ & $>0,05$ \\
\hline Three somersaults forward, s & $4,2 \pm 0,16$ & $4,33 \pm 0,12$ & $>0,05$ \\
\hline Shuttle run 4x10m with cones, s & $12,35 \pm 0,27$ & $13,02 \pm 0,18$ & $>0,05$ \\
& & & \\
\hline Shuttle run 3x10M, s & $9,2 \pm 0,22$ & $9,3 \pm 0,18$ \\
\hline
\end{tabular}

Both groups were assessed the level of rhythm sense development, using a computer test " Got Rhythm?", located on the Internet resource www.concerthotels. The test works as follows: the metronome sets the reference rhythm, the subject simultaneously with the metronome signal presses the "Space" key on the computer keyboard or touches the screen of the touch phone. After 5 taps, the sound of the metronome disappears, the subject must perform another 20 taps in the same rhythm. The program measures the time deviation of each click from the set reference rhythm. The sense of rhythm is evaluated in conventional units on a scale from 0 to 1000 units. A better sense of rhythm corresponds to a larger number of units. This test showed no difference between EG and CG. The test results are shown in table 2.

Testing of children did not reveal statistically significant differences between EG and CG, not on one of the tests and confirmed the correct selection of children in groups.

\section{Results and discussion}

The analysis of the tests results is particular interesting. It was produced in the following way. Lists of children in groups were ranked according to the results of the rhythm test. The best results correspond to the beginning of the list, the worst to the end. Based on the results obtained, graphs were constructed. The abscissa axis shows the number of the test subject in the list, and the ordinate axis shows the test result. 


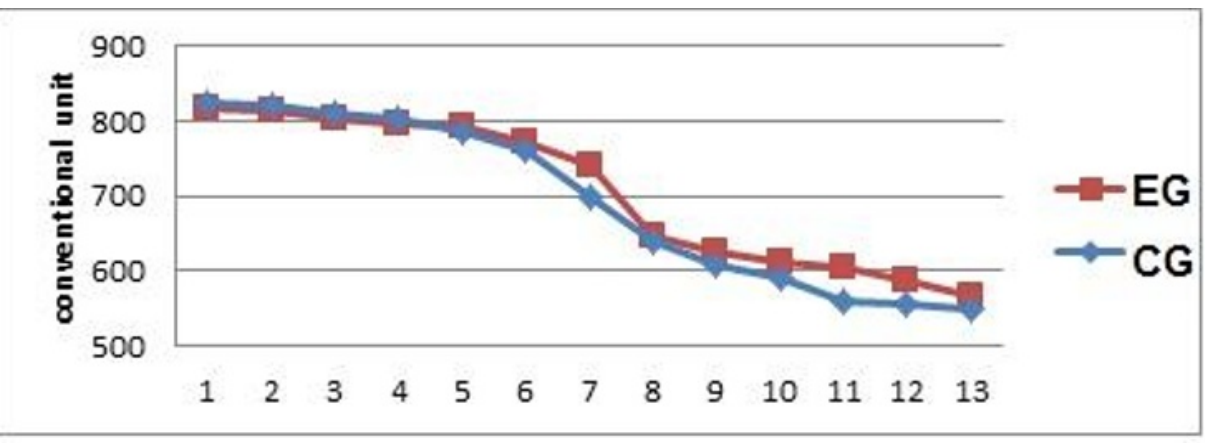

Fig. 1. Results of the "sense of rhythm" test.

The graph of the "sense of rhythm" test Fig.1 shows that children in groups, according to the level of rhythm sense development,are divided into two subgroups. Children with a well-developed sense of rhythm (the first 7) and with a less developed sense of rhythm (the other 6).

Pedagogical observation reveales a connection between the sense of rhythm and the results of HC classes. The children who have the best results in mastering hand-to-hand combat are all in the subgroup with a high level of rhythm sense development.

Graphs of all tests are constructed in accordance with the resulting ranked list. Visual analysis of the graphs provides additional information.

The graphs show that children in the first half of the list have the best performance in almost all tests, except for the "Passing the ball from the wall" test. For example, here is the graph of the test "Running $30 \mathrm{~m}$ " Fig. 2, the rest have a similar appearance.

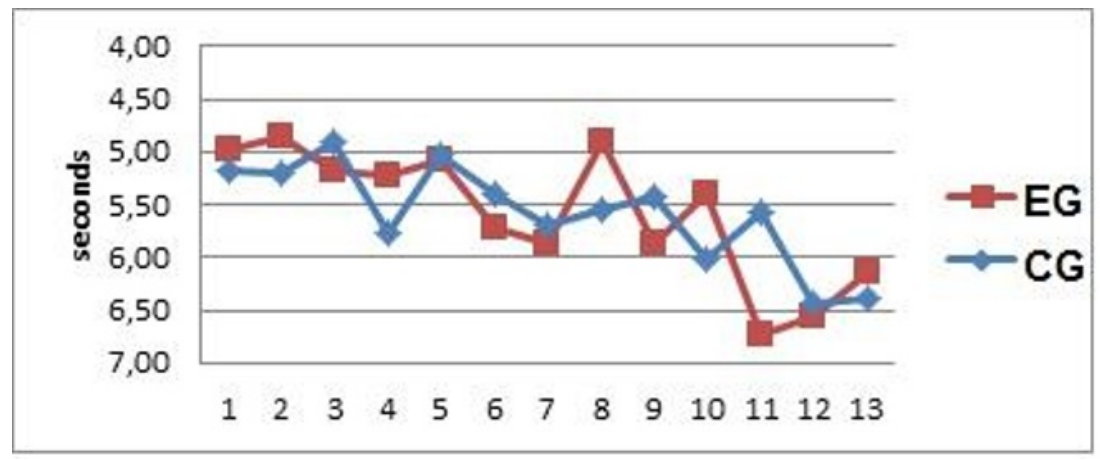

Fig. 2. The results of the test "Running $30 \mathrm{~m} »$.

This confirms the pedagogical observation about the greater overall development of children with a good sense of rhythm and the idea that the sense of rhythm has a motor nature $[6,8]$.

Graph of the test "Passing the ball from the wall" Fig.3 this is the only chart that does not have decrease with the growth of the list number and is located almost horizontally.

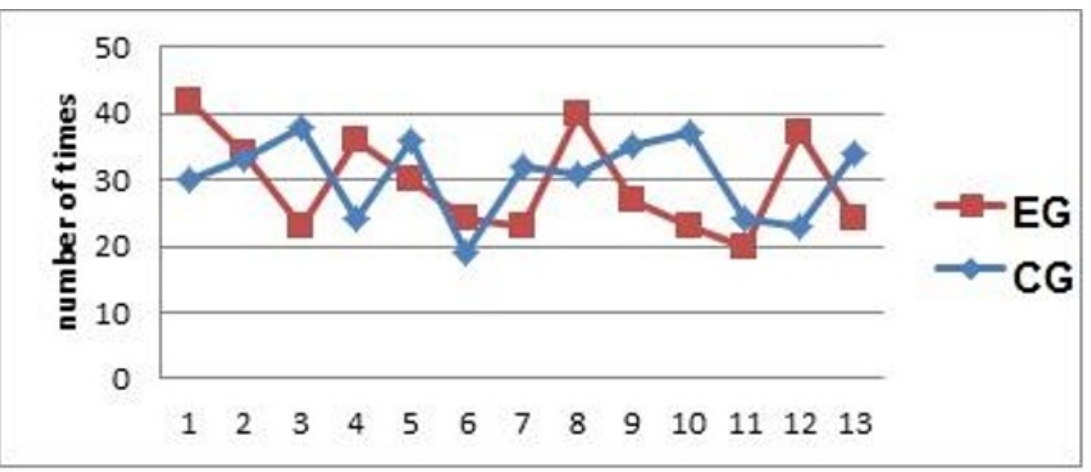

Fig. 3. Results of the test " Passing the ball from the wall».

This suggests that the test results are independent of both the rhythm sense and the level of physical qualities development of children in groups. The tests used a light ball, which all children equally coped with. Throws were performed mainly by hands, without connecting the body and legs. Hand movements in humans have a high degree of independence, both among themselves and from the movements of the rest body. While performing the test 
children worked not by rhythmizing movements, but by using a visual analyzer, reacted to a moving object, a ball bouncing off a wall.

Interesting results are given by the analysis of the Cross test graph in Fig. 4.

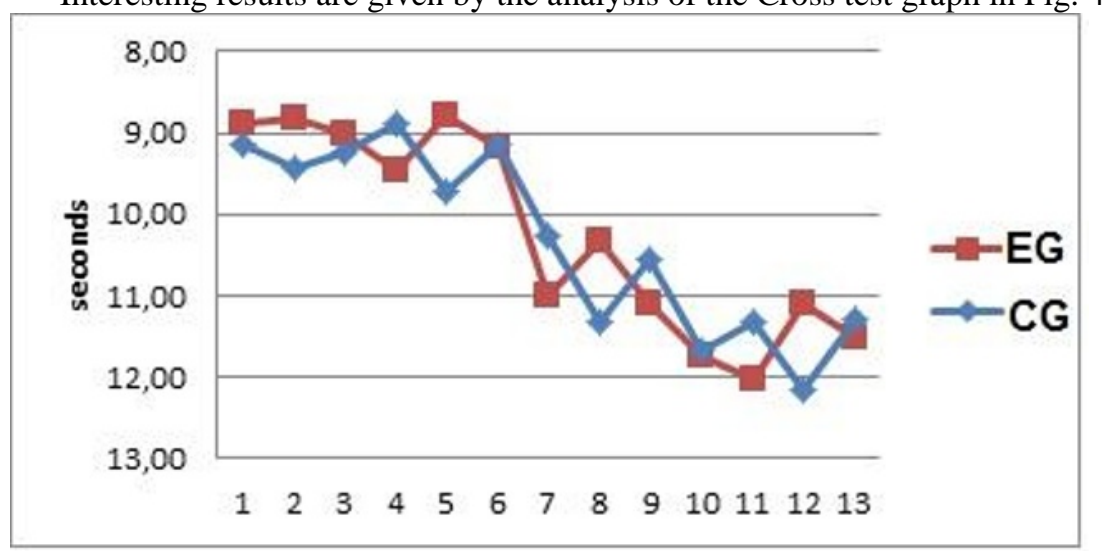

Fig. 4. The results of the test "Cross"

The graph of this test is similar in form to the graph of the rhythm test. On this basis, we can make an assumption about the dependence of the results in this test on the development of a rhythm sense.

At the end of the pedagogical experiment, repeated testing was conducted. It showed that a statistically significant difference between EG and CG appeared only in three tests: "sense of rhythm", "Cross " and "Passing the ball from the wall". In tests for general physical fitness, in coordination tests based on locomotor movements ("3x10m Shuttle run" and "4x10m Shuttle run with cones"), as well as in the" Three somersaults " test, no differences were found between the experimental and control groups at the end of the experiment. This indicates the rhythmic independence of the results in these tests.

The assumption about the rhythm dependence of the results in the "Cross" test was confirmed. This is of particular importance for the HC. The movements used for the "Cross" test are similar in structure to the basic movements used in hand-to-hand combat techniques, and in wrestling, these are entrances to throws with a turn, sweeps, etc. Moves are the foundation on which a match in $\mathrm{HC}$ is built. The research shows that the quality of their performance depends on the sense of rhythm.

Explaining the growth of the results in the test "Passing the ball from the wall" required additional research. Testing before the start of the pedagogical experiment revealed its rhythm independence.

Pedagogical observation of children showed that there were changes in the technique of performing the test "Passing the ball from the wall". In the test, before the experiment, children performed passes by working their hands while standing still. It forced them to tilt the body to the side to catch the ball that did not exactly bounce, and the next throw, only after returning to the starting position. There was a loss of time to return to the starting position.

In tests, after the experiment was completed, they began to use Shuttle movements to the right and left, with a flat body, to compensate for errors. This allowed you to pass immediately after catching the ball. There were no such changes in the test technique in the CG.

The survey of EG children revealed that in addition to the visual analyzer, they included the auditory one. The ball hitting the wall is clearly audible, and additional information from the auditory analyzer allowed children to rhythmize their movements, thereby increasing their frequency.

The auditory analyzer can be used effectively during HC, giving the fighter important additional information about the opponent. During the battle, the enemy's movements are often clearly heard, his breathing is heard, and any contact interaction is accompanied by sounds.

\section{Conclusions}

The pedagogical experiment showed the necessity and importance of including in the training process in HC work on the rhythm sense development.

The study revealed the rhythm dependence of the movements skill during a fight in HC. This is the foundation on which the entire fight is built. Work on the rhythm sense led to the inclusion of an auditory analyzer use during the "Passing the ball from the wall" test. It is quite logical that the use of an auditory analyzer can be transferred to the conduct of a match in the HC.

The quality of performing technical actions in hand-to-hand combat, during the experiment, in the EG increased to a greater extent than in the CG. However, additional research is required on the relationship between the sense of rhythm and the level of technical readiness in the HC.

Based on the results of the study, we can make an assumption about the structure of human CA. Nikolai Bernstein also distinguished between "manual" and "bodily" dexterity [1]. Research suggests that dexterity (CA) can be divided into manual, bodily, and locomotor. They must be evaluated and developed in a differentiated way. All this requires further research. 


\section{References}

1. N. A. Bernstein, On dexterity and its development (FIS, Moscow, 2001)

2. V. L. Botyaev, Theory and practice of physical culture, 7, 58-61 (2012)

3. V. E. Zankovets, Encyclopedia of testing (Sport, Moscow, 2016)

4. V. I. Lyakh, Coordination abilities: diagnostics and development (TVT Division, Moscow, 2006)

5. Hand-to-hand combat. Sample program of sports training for children's and youth sports schools, specialized children's and youth schools of the Olympic reserve (Sovetsky sport, Moscow, 2004)

6. A.S. Kuznetsov, Z.M. Kuznetsova, Russian Journal of Physical Education and Sport, 14(4), 5-7 (2019). DOI: 10.14526/20704798-2019-14-4-5-7

7. S. E. Toksinov, I. U. Gorskaya, A. S. Dmitrienko, Scientific notes of the University named after P. F. Lesgaft, 12(178), 305-310 (2019)

8. B. V. Shilakin, A.V. Zakharov, A.D. Kalinenko, Scientific notes of the University named after P. F. Lesgaft, 5(159), 303-307 (2018) 